\title{
Erratum to: Nonlocal fourth-order Kirchhoff systems with variable growth: low and high energy solutions
}

\author{
Ghasem A. Afrouzi ${ }^{1}$ - M. Mirzapour ${ }^{2}$. \\ Vicenţiu D. Rădulescu ${ }^{3,4}$
}

Published online: 9 February 2016

(C) Universitat de Barcelona 2016

\section{Erratum to: Collect. Math. DOI 10.1007/s13348-014-0131-x}

The goal of this erratum is to correct a mistake that appears in the assumption $\left(M_{2}\right)$ in the original article. In the correct version, the hypothesis $\left(M_{2}\right)$ should be removed. In such a case, we restate the following assumption:

$\left(M_{1}\right)$ There exist $m_{2} \geq m_{1}>0$ and $\alpha>1$ such that $m_{1} t^{\alpha-1} \leq M(t) \leq m_{2} t^{\alpha-1}$, for all $t \in \mathbb{R}^{+}$.

We point out that the original assumption $\left(M_{1}\right)$ implies $\alpha_{1}=\alpha_{2}$, so we rename constant $\alpha$. In conditions $\left(F_{2}\right)$ and $\left(F_{5}\right)$, we replace $\beta$ by $\alpha$.

The correct statement of Lemma 3.2 is the following.

The online version of the original article can be found under doi:10.1007/s13348-014-0131-x.

$\varangle \quad$ Vicenţiu D. Rădulescu

vicentiu.radulescu@imar.ro

Ghasem A. Afrouzi

afrouzi@umz.ac.ir

M. Mirzapour

mirzapour@stu.umz.ac.ir

1 Department of Mathematics, Faculty of Mathematical Sciences, University of Mazandaran, Babolsar, Iran

2 Farhangian University, Tehran, Iran

3 Department of Mathematics, Faculty of Sciences, King Abdulaziz University, P.O. Box 80203, 21589 Jeddah, Saudi Arabia

4 Institute of Mathematics "Simion Stoilow" of the Romanian Academy, P.O. Box 1-764, 014700 Bucharest, Romania 
Lemma 3.2 Let $\left(u_{n}, v_{n}\right)$ be a Palais-Smale sequence for the Euler-Lagrange functional $J$. Assume that conditions $\left(M_{1}\right),\left(F_{2}\right)$ are satisfied and

$$
m_{1} \theta_{1}\left(p^{-}\right)^{\alpha-1}>\alpha m_{2}, \quad m_{1} \theta_{2}\left(q^{-}\right)^{\alpha-1}>\alpha m_{2} .
$$

Then the sequence $\left(u_{n}, v_{n}\right)$ is bounded.

In the proof of Lemma 3.2, by hypotheses $(0.1),\left(M_{1}\right)$ and $\left(F_{2}\right)$, we can write for $n$ large enough

$$
\begin{aligned}
c_{7} \geq & J\left(u_{n}, v_{n}\right) \geq \frac{m_{1}}{\alpha}\left(\int_{\Omega} \frac{1}{p(x)}\left|\Delta u_{n}\right|^{p(x)} d x\right)^{\alpha}-\int_{\Omega} \frac{u_{n}}{\theta_{1}} \frac{\partial F}{\partial u}\left(x, u_{n}, v_{n}\right) d x \\
& +\frac{m_{1}}{\alpha}\left(\int_{\Omega} \frac{1}{q(x)}\left|\Delta v_{n}\right|^{q(x)} d x\right)^{\alpha}-\int_{\Omega} \frac{v_{n}}{\theta_{2}} \frac{\partial F}{\partial v}\left(x, u_{n}, v_{n}\right) d x-c_{8},
\end{aligned}
$$

where $c_{8}$ is a positive constant. Therefore

$$
\begin{aligned}
c_{7} \geq & J\left(u_{n}, v_{n}\right) \\
\geq & \frac{m_{1}}{\alpha}\left(\int_{\Omega} \frac{1}{p(x)}\left|\Delta u_{n}\right|^{p(x)} d x\right)^{\alpha}-\frac{m_{2}}{\theta_{1}}\left(\int_{\Omega} \frac{1}{p(x)}\left|\Delta u_{n}\right|^{p(x)} d x\right)^{\alpha-1} \int_{\Omega}\left|\Delta u_{n}\right|^{p(x)} d x \\
& +\frac{1}{\theta_{1}} D_{1} J\left(u_{n}, v_{n}\right)\left(u_{n}\right) \\
& +\frac{m_{1}}{\alpha}\left(\int_{\Omega} \frac{1}{q(x)}\left|\Delta v_{n}\right|^{q(x)} d x\right)^{\alpha}-\frac{m_{2}}{\theta_{2}}\left(\int_{\Omega} \frac{1}{q(x)}\left|\Delta v_{n}\right|^{p(x)} d x\right)^{\alpha-1} \int_{\Omega}\left|\Delta v_{n}\right|^{p(x)} d x \\
& +\frac{1}{\theta_{2}} D_{2} J\left(u_{n}, v_{n}\right)\left(v_{n}\right)-c_{8} \\
\geq & \left(\frac{m_{1}}{\alpha}-\frac{m_{2}}{\theta_{1}\left(p^{-}\right)^{\alpha-1}}\right)\left(\int_{\Omega}\left|\Delta u_{n}\right|^{p(x)} d x\right)^{\alpha}+\left(\frac{m_{1}}{\alpha}-\frac{m_{2}}{\theta_{2}\left(q^{-}\right)^{\alpha-1}}\right)\left(\int_{\Omega}\left|\Delta v_{n}\right|^{q(x)} d x\right)^{\alpha} \\
& -\frac{1}{\theta_{1}}\left\|D_{1} J\left(u_{n}, v_{n}\right)\right\|_{*, p(x)}\left\|u_{n}\right\|-\frac{1}{\theta_{2}}\left\|D_{2} J\left(u_{n}, v_{n}\right)\right\|_{*, q(x)}\left\|v_{n}\right\|-c_{8} .
\end{aligned}
$$

Now, we suppose that the sequence $\left(u_{n}, v_{n}\right)$ is not bounded. Without loss of generality, we may assume $\left\|u_{n}\right\|_{p(x)} \geq\left\|v_{n}\right\|_{q(x)}$. Therefore, for $n$ large enough so that $\left\|u_{n}\right\|_{p(x)}>1$, we obtain

$$
\begin{aligned}
c_{7} \geq & \left(\frac{m_{1}}{\alpha}-\frac{m_{2}}{\theta_{1}\left(p^{-}\right)^{\alpha-1}}\right)\left\|u_{n}\right\|_{p(x)}^{\alpha p^{-}} \\
& -\left(\frac{1}{\theta_{1}}\left\|D_{1} J\left(u_{n}, v_{n}\right)\right\|_{*, p}+\frac{1}{\theta_{2}}\left\|D_{2} J\left(u_{n}, v_{n}\right)\right\|_{*, q}\right)\left\|u_{n}\right\|_{p(x)} .
\end{aligned}
$$

But this cannot hold since $\alpha p^{-}>p^{-}>1$. Hence, $\left(u_{n}, v_{n}\right)$ is bounded.

Theorem 3.1 and Lemma 3.3 remain unchanged. However, Theorems 3.4, 4.1, 4.2 and Lemmas 3.2, 3.3 need to be stated without assumption $\left(M_{2}\right)$. Hypothesis $(0.1)$ should be also added in the statement of Theorems 3.4 and 4.1. The proofs of Theorems 3.4, 4.1 and 4.2 are similar to the original proofs, but replacing $\beta$ by $\alpha$. 